NISTIR 89-4028

NEW NIST PUBLICATION

November 1989

\title{
A GYPSUM \\ WALLBOARD FORMALDEHYDE SORPTION MODEL
}

\section{Samuel Silberstein}

U.S. DEPARTMENT OF COMMERCE Natlonal Institute of Standards and Technology Natlonal Englneorlng Laboratory Center for Bullding Technology Bullding Environment Divislon Galthersburg, MD 20899

U.S. DEPARTMENT OF COMMERCE Robert A. Mosbacher, Secretary NATIONAL INSTITUTE OF STANDARDS AND TECHNOLOGY

Raymond G. Kammer, Actlng Director

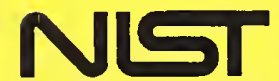





\title{
A GYPSUM WALLBOARD FORMALDEHYDE SORPTION MODEL
}

\section{Samuel Silberstein}

\author{
U.S. DEPARTMENT OF COMMERCE \\ National Institute of Standards \\ and Technology \\ Natlonal Englnoerlng Laboratory \\ Center for Bullding Technology \\ Bullding Environment Division \\ Galthersburg, MD 20899
}

November 1989

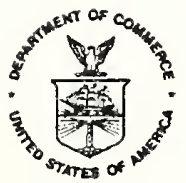

U.S. DEPARTMENT OF COMMERCE Robert A. Mosbacher, Secretary NATIONAL INSTITUTE OF STANDARDS AND TECHNOLOGY

Raymond G. Kammer, Acting Dlrector 

Abstract

Gypsum wallboard was shown to absorb formaldehyde in a prototype house and in a measuring chamber, as reported previously by researchers at Oak Ridge National Laboratory (ORNL). Also as reported previously, formaldehyde concentrations attained equilibrium in two phases in response to a change in the air exchange rate or to the removal of the formaldehyde source. A rapid initial phase was followed by a slow phase lasting several days. A formaldehyde sorption model that accounts for the biphasic concentration pattern is presented here. Experiments for testing the predictability of the model are proposed.

Key words: Formaldehyde; gypsum; indoor air quality; modeling; pollutant; sorption; source-sink; wallboard. 

$a=$ normalized coefficient in equations for $C(t)$.

$A_{i}=$ exposed surface area of the $i^{\text {th }}$ pressed-wood product (subscript), or of gypsum wallboard (no subscript), $\mathrm{m}^{2}$.

$a_{\mathrm{g}}=$ normalized coefficient in equations for $\mathrm{G}(\mathrm{t})$.

$\alpha_{i}$ and $\beta_{i}$ are linear regression coefficients in the equations: $E_{i}=\alpha_{i}$ $\beta_{i} \cdot \mathrm{C}, \mathrm{mg} / \mathrm{m}^{2} \cdot \mathrm{h}$ and $\mathrm{m} / \mathrm{h}$, respectively.

$C^{*}=C(t)=$ formaldehyde concentration at time $t, \mathrm{mg} / \mathrm{m}^{3}$.

$\mathrm{C}_{\text {ext }}=$ external formaldehyde concentration, $\mathrm{mg} / \mathrm{m}^{3}$.

$\mathrm{C}_{\infty}^{\prime}=$ formaldehyde concentration at time $\infty$ in the absence of gypsum wallboard, $\mathrm{mg} / \mathrm{m}^{3}$.

$\underline{e}=$ formaldehyde emission rate of permeation tubes, $\mathrm{mg} / \mathrm{h}$.

$\tilde{\mathrm{e}}=\mathrm{e}+\Sigma \mathrm{A}_{\mathrm{i}} \cdot \alpha_{\mathrm{i}}, \mathrm{mg} / \mathrm{h}$.

$E_{i}=$ formaldehyde emission rate per unit area of the $i^{\text {th }}$ pressed-wood product, $\mathrm{mg} / \mathrm{m}^{2} \cdot \mathrm{h}$.

$F^{*}=F(t)=$ formaldehyde content of the measuring chamber at time $t$, $m$.

$\phi_{1}=$ slow decay constant, $\mathrm{h}^{-1}$.

$\phi_{2}=$ fast decay constant, $\mathrm{h}^{-1}$.

$\mathrm{G}^{*}=\mathrm{G}(\mathrm{t})=$ formaldehyde content of the gypsum wallboard per $1 \mathrm{~m}$ area at time $t, \mathrm{mg} / \mathrm{m}^{2}$.

$I=\underset{\sim}{i} r$ exchange rate, $h^{-1}$.

$I^{\prime}=\tilde{e} /\left(V \cdot C_{\infty}\right), h^{-1}$.

$\overline{\mathrm{I}}=\mathrm{I}+\Sigma \mathrm{L}_{\mathrm{i}} \cdot \beta_{\mathrm{i}}, \mathrm{h}^{-1}$.

$\mathrm{K}=\mathrm{G}_{\infty} / \mathrm{C}_{\infty}, \mathrm{m}$.

$\mathrm{k}_{1}=$ model constant, $\mathrm{m} / \mathrm{h}$.

$\mathrm{k}_{1}^{\prime}=\mathrm{k}_{1}{ }^{\prime} \cdot \mathrm{V} / \mathrm{A}, \mathrm{h}^{-1}$.

$\mathrm{k}_{2}=$ model constant, $\mathrm{h}^{-1}$.

$k_{\mathrm{d}}=$ first order rate constant for irreversible formaldehyde removal per unit area of gypsum wallboard, $h^{-1}$.

$\mathrm{L}_{i}=\mathrm{A}_{i} / \mathrm{V}=$ loading of the $i^{\text {th }}$ pressed-wood product (subscript), or of gypsum wallboard (no subscript) $\mathrm{m}^{-1}$.

$\mathrm{RH}=$ relative humidity, 8 .

$S=\Sigma A_{i} \cdot E_{i}, m g / h$.

$t=$ time, $h$.

$\tau_{i}=\phi_{i}^{-1}=$ time constant, h.

$\mathrm{V}=$ volume, $\mathrm{m}^{3}$.

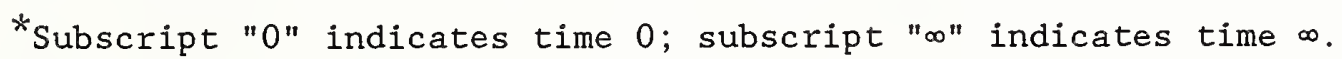

\section{INTRODUCTION}

Pressed-wood products are a major source of formaldehyde in indoor environments. Several models successfully predict equilibrium indoor formaldehyde levels from knowledge of the emission rate of pressed-wood product emitters [1-13]. However, the formaldehyde concentration may take days to achieve equilibrium because of the presence of sinks in houses, most notably gypsum wallboard. In order to successfully predict 
indoor formaldehyde concentrations, models must describe non-equilibrium concentrations in the presence of wallboard.

Matthews et al. [12] studied formaldehyde sorption at Oak Ridge National Laboratory (ORNL). The formaldehyde concentration in smallscale environmental chambers containing pressed-wood products was monitored while formaldehyde was injected and absorbed by gypsum wallboard for several days, followed by several days of desorption. The ORNL researchers observed a biphasic formaldehyde concentration profile, in which the concentration rose (fell) abruptly within the first 2 hours of absorption (desorption) followed by a slow approach to equilibrium. A similar pattern was observed at the National Institute of Standards and Technology (NIST) in response to changes in the air exchange rate (Figure 1) of a prototype house containing pressed-wood products as the formaldehyde source. A similar profile was also observed when formaldehyde was injected at a constant rate into a measuring chamber containing an uncut wallboard specimen (Figure 2), and after injection was stopped.

The ORNL researchers described concentration for times greater than 2 hours by an exponential term with a time constant $\tau \approx 2.5-3$ days for various ratios of air exchange rate (I) to wallboard loading ( $\mathrm{L}=\mathrm{A} / \mathrm{V}$ where $A=$ wallboard area and $V=$ volume). They did not suggest how the coefficients of their equation can be modified to account for conditions different from those used in their experiments.

Sorption of other gases, for example $\mathrm{NO}_{2}$, NO, and $\mathrm{SO}_{2}$, by home furnishings has been modeled on the basis of mass balance [14-16]. Deposition velocity of $\mathrm{NO}_{2}$ depended upon temperature, $\mathrm{RH}$, and mixing. The present report uses a similar approach to derive a formaldehyde sorption model, taking account of the differences from $\mathrm{NO}_{2}$ and $\mathrm{SO}_{2}$ sorption. Once absorbed, $\mathrm{NO}_{2}$ and $\mathrm{SO}_{2}$ are not emitted. In contrast, Matthews et al. estimated that 90-95\% of the formaldehyde absorbed by wallboard is later reemitted, implying that it does not react irreversibly in air or wallboard to an appreciable extent [12]. The outdoor concentration pattern, especially of $\mathrm{NO}_{2}$, may strongly influence its indoor concentration, whereas outdoor formaldehyde concentrations are generally low compared to those in buildings containing formaldehyde emitters [1-2].

The assumptions used in modeling formaldehyde sorption are discussed in Section 2, followed by presentation of the model in terms of model constants which should enable the model to be used at different air exchange rates, pressed-wood product and wallboard areas, and room volumes. The formaldehyde concentration is derived from the model. The mathematics of deriving the model constants from quantities obtained by measuring chamber experiments is described in Section 3. Experiments for calculating the model constants, testing the assumptions underlying the model, extending the model to different combinations of temperature and relative humidity $(\mathrm{RH})$, and validating the model in houses are described in Section 4. The preliminary experiments used to obtain the results shown in Figures 1-2 are described in Appendix A. 


\section{DERIVATION OF A GYPSUM WALLBOARD FORMALDEHYDE SORPTION MODEL}

\subsection{Assumptions}

A simplified picture of formaldehyde sorption in a room or measuring chamber containing gypsum wallboard is used to derive sorption models. Formaldehyde and its binding sites on or within wallboard are assumed to be uniformly distributed per unit surface area. The dependence of distribution on depth into the wallboard is unknown. The affinity of the binding sites, which may include trapped water, to formaldehyde is assumed to be constant. It is assumed that formaldehyde reacts irreversibly within or on the surface of wallboard to some unknown product(s) in a first-order reaction. Assuming binding site affinity and reaction rate to be constant means using weighted average values in an analogous manner to using "center of mass" in dynamics problems. A general model would allow for irreversible reaction within room air as well, but the rate of such a reaction is assumed to be negligible in the present model.

Throughout the modeling, it is assumed that formaldehyde concentration (C), wallboard content per unit area (G), and emission rate (e) are small enough so that sorption is first-order, that is that the absorptive capacity of the wallboard is not approached. Assume first that $G=0 \mathrm{mg} / \mathrm{m}^{2}, I=0 \mathrm{~h}^{-1}$, and $\mathrm{e}=0 \mathrm{mg} / \mathrm{h}$. Then the formaldehyde entry rate into the wallboard is assumed to be proportional to $\mathrm{C}$ and $\mathrm{A}$ with proportionality constant $k_{1}: G=k_{1} \cdot C \cdot A$ and by mass balance, $C=k_{1} \cdot C \cdot L$. Assume instead now that $C=0 \mathrm{mg} / \mathrm{m}^{3}$. Then the emission rate from and rate of irreversible removal by wallboard are both assumed to be proportional to the formaldehyde content of the wallboard with proportionality constants $k_{2}$ and $k_{d}$, respectively: $G=-\left(k_{2}+k_{d}\right) \cdot G$ and by mass balance $C=k_{2} \cdot L \cdot G$.

Assuming that sorption is first order means that the rate-limiting step of sorption is first-order. In particular, if diffusion is the rate-limiting step, then it obeys Fick's diffusion law, implying that it is not obstructed to the extent that the rate of absoption is independent of formaldehyde concentration (zero order kinetics). Suppose, contrary to assumption, that absorption were zero order. If the formaldehyde exit rate were also zero order, then the resulting formaldehyde concentration profile would not be biphasic, but a simple exponential expression, contradicting observations at ORNL and NIST. If the formaldehyde exit rate were first order, then the resulting concentration profile would be biphasic. The values of the $\phi_{i}$ (the negative of the eigenvalues for the sorption model) would be given by $I$ and $k_{2}+k_{d}$. I is estimated to be approximately $1 \mathrm{~h}^{-1}$. However, as shown in Appendix $\mathrm{A}$, the values of the $\phi_{i}$ were determined to be 0.0076 and $3 \mathrm{~h}^{-1}$ for the sorption data shown in Figure 2. Therefore, zero order kinetics does not seem to be consistent with the results of this experiment. 


\subsection{The Gypsum-Board Formaldehyde Sorption Model}

In general, $C, G$, e, and I are not necessarily zero. On the basis of the assumptions above, the following two-equation sorption model is predicted to describe $C$ and $G$ at fixed temperature and $\mathrm{RH}$ :

$$
\begin{aligned}
& C=-\left(I+k_{1} \cdot L\right) \cdot C+\left(k_{2} \cdot L\right) \cdot G+e / V \\
& G=k_{1} \cdot C-\left(k_{2}+k_{d}\right) \cdot G
\end{aligned}
$$

It is important to note that at fixed temperature and $\mathrm{RH}, \mathrm{k}_{1}, \mathrm{k}_{2}$, and $k_{d}$ are invariant in $A$ and $V$, and hence in $L$ as well. The physical meaning of $k_{1}$ and $k_{2}$ is not specified in the model. A very useful invariant is $\mathrm{K}$, the equilibrium wallboard-formaldehyde content per unit area per unit airborne equilibrium concentration $\left(1 \mathrm{mg} / \mathrm{m}^{3}\right)$. At $t=\infty, G$ $=0 \mathrm{mg} /\left(\mathrm{m}^{2} \cdot \mathrm{h}\right), \mathrm{C}=\mathrm{C}_{\infty}$, and $\mathrm{G}=\mathrm{G}_{\infty}$ in Equation 2.2:

$$
\mathrm{K}=\mathrm{G}_{\infty} / \mathrm{C}_{\infty}=\mathrm{k}_{1} /\left(\mathrm{k}_{2}+\mathrm{k}_{\mathrm{d}}\right)
$$

Matthews et al. determined $\mathrm{K}$ to be $23 \mathrm{mg} / \mathrm{m}^{2}$ per unit formaldehyde concentration for the wallboard specimens they tested [12]. Another important constant, $\mathrm{K} \cdot \mathrm{L}$, is a partitioning constant; it is the ratio of the formaldehyde content of the wallboard to the content of room air at equilibrium. Unlike $\mathrm{K}, \mathrm{K} \cdot \mathrm{L}$ is not invariant in $\mathrm{A}$ and $\mathrm{V}$ but depends on their ratio. For a typical value of $\mathrm{L}=1.6 \mathrm{~m}^{-1}$ and the value of $\mathrm{K}$ determined by ORNL, $K \cdot L=37$; the alr contains less than 38 of the formaldehyde in the room.

2.1. Sorption Model, Formaldehyde Concentration, and Gypsum Wallboard Content when Pressed-Wood Products are Present

Equation 2.1 must be modified for the presence of pressed wood products. It has been shown repeatedly that the formaldehyde emission rate of pressed-wood products is linear in formaldehyde concentration, with negative slope, as a consequence of Fick's law of diffusion [1-13]. Therefore, if there are $n$ pressed-wood products, then $E_{i}$, the formaldehyde emission rate per unit area $\left(A_{i}\right)$ of the $i^{\text {th }}$ board, is given by:

$$
E_{i}=\alpha_{i}-\beta_{i} \cdot C \text { for } C \leq \alpha_{i} / \beta_{i}
$$

(The constants $\alpha_{i}$ and $\beta_{i}$ are determined by linear regression analysis of measured concentrations vs. surface emission rates, as described elsewhere $[2,8,13]$.$) Then \mathrm{S}$, the formaldehyde generation rate, is given by:

$$
\mathrm{S}=\Sigma \mathrm{A}_{i} \cdot \alpha_{i}-\left(\Sigma \mathrm{A}_{i} \cdot \beta_{i}\right) \cdot \mathrm{C} \text { for } \mathrm{C} \leq \min \left(\alpha_{i} / \beta_{i}\right)
$$

(At concentrations $C>\min \left(\alpha_{i} / \beta_{i}\right)$, the weak formaldehyde emitters may cease to emit altogether and become sinks [17-18].) Note that the first 
term on the right of Equation 2.5 is constant while the second term is linear in $C$. Incorporating $S$ into Equation 2.1, with $L_{i}=A_{i} / V$ :

$$
C=-\left(I+k_{1} \cdot L\right) \cdot C+k_{2} \cdot L \cdot G+e / V+\Sigma L_{i} \cdot \alpha_{i}-\left(\Sigma L_{i} \cdot \beta_{i}\right) \cdot C
$$

Rearranging terms:

$$
C=-\left(I+k_{1} \cdot L+\Sigma L_{i} \cdot \beta_{i}\right) \cdot C+k_{2} \cdot L \cdot G+\left(e+\Sigma A_{i} \cdot \alpha_{i}\right) / V
$$

Formally, Equation 2.7 is the same as Equation 2.1 , with e replaced by $\tilde{e}$ $=e+\Sigma A_{i} \cdot \alpha_{i}$ and $I$ replaced by $\tilde{I}=I+\Sigma L_{i} \cdot \beta_{i}$.

3. DETERMINATION OF FORMALDEHYDE CONCENTRATION AND GYPSUM WALLBOARD CONTENT

Let $-\phi_{1}$ and $-\phi_{2}$ be the eigenvalues for the system of equations 2.1 and 2.2. Assume that $C_{\text {ext }}$, the external formaldehyde concentration, is negligible. $G_{\infty}$, the equilibrium gypsum wallboard formaldehyde content, is given below by Equation 4.11. Assume that a disturbance occurs at time 0 , for example $I$ or $e$ is changed. Then, at time $t, C$ and $G$ are given by:

$C(t)=C_{\infty}+\left(C_{0}-C_{\infty}\right) \cdot\left(a \cdot \exp (-\phi 1 \cdot t)+(1-a) \cdot \exp \left(-\phi_{2} \cdot t\right)\right)$

$G(t)=G_{\infty}+\left(G_{0}-G_{\infty}\right) \cdot\left(a_{g} \cdot \exp \left(-\phi_{1} \cdot t\right)+\left(1-a_{g}\right) \cdot \exp \left(-\phi_{2} \cdot t\right)\right)$

Net absorption occurs when $\mathrm{C}_{0}<\mathrm{C}_{\infty}$, net desorption when $\mathrm{C}_{0}>\mathrm{C}_{\infty}$.

Typically $\phi_{2} \gg \phi_{1}$. If $t \gg \phi_{2}{ }^{-1}$, then $\exp \left(-\phi_{2} \cdot t\right) \approx 0$.

Furthermore, as is shown below, $a_{g} \approx 1$. Thus, when $\phi_{2} \gg \phi_{1}$ and $t \gg$ $\phi_{2}{ }^{-1}$, Equations 3.1-3.2 can be replaced by Equations 3.3-3.4:

$$
\begin{aligned}
& C(t) \approx C_{\infty}+\left(C_{0}-C_{\infty}\right) \cdot a \cdot \exp (-\phi 1 \cdot t) \\
& G(t) \approx G_{\infty}+\left(G_{0}-G_{\infty}\right) \cdot \exp \left(-\phi_{1} \cdot t\right)
\end{aligned}
$$

For notational convenience, assume that pressed-wood products are absent. To obtain eigenvalues and coefficients when pressed-wood products are present, replace each occurrence of $e$ by $e$, and of $I$ by $\tilde{I}$ throughout this section.

Define $\mathrm{k}_{1}{ }^{\prime}=\mathrm{k}_{1} \cdot \mathrm{L}$, and $\delta$ (the "discriminant") by:

$$
\delta^{2}=\left(I+k_{1}{ }^{\prime}+k_{2}+k_{d}\right)^{2}-4 \cdot I \cdot k_{2}-4 \cdot k_{d} \cdot\left(I+k_{1}{ }^{\prime}\right)
$$

Then:

$$
\begin{aligned}
& \phi_{1}=\left(I+k_{1}^{\prime}+k_{2}+k_{d}-\delta\right) / 2 \\
& \phi_{2}=\left(I+k_{1}^{\prime}+k_{2}+k_{d}+\delta\right) / 2=\phi_{1}+\delta
\end{aligned}
$$

I and $C_{\infty}{ }^{\prime}$, the equilibrium formaldehyde concentration in the 
absence of wallboard, or equivalently in the presence of wallboard for which $k_{\mathrm{d}}=0 \mathrm{~h}^{-1}$, are related by:

$$
\mathrm{e}=\mathrm{I} \cdot \mathrm{C}_{\infty} \cdot \cdot \mathrm{V}
$$

If $C_{\infty}$ is substituted for $C_{\infty}$ ' in Equation 3.8, then $I^{\prime}$, the "apparent air exchange rate" obtained by using formaldehyde as a tracer gas, must be substituted for I to maintain the same relationship:

$$
e=I^{\prime} \cdot C_{\infty} \cdot V
$$

It follows that $I \leq I^{\prime}$ and:

$$
C_{\infty} / C_{\infty}^{\prime}=I / I^{\prime}
$$

Each of the ratios in Equation 3.10 is equal to 1 if and only if $k_{d}=0$ $\mathrm{h}^{-1}$. The normalized coefficients of Equations 3.1 and 3.3 , $a$ and $1-a$, are given by:

$$
\begin{aligned}
& a=\left(\phi_{2}-I^{\prime}\right) /\left(\phi_{2}-\phi_{1}\right) \\
& 1-a=\left(I^{\prime}-\phi_{1}\right) /\left(\phi_{2}-\phi_{1}\right)
\end{aligned}
$$

Rearranging Equation 3.11, one obtains an expression for $\phi_{2}$ in terms of experimentally derived quantities:

$$
\phi_{2}=\left(I^{\prime}-\phi_{1} \cdot a\right) /(1-a)
$$

For $\phi_{2} \gg \phi_{1}$ :

$$
\begin{aligned}
& a \approx 1-I^{\prime} / \phi_{2} \\
& 1-a \approx I^{\prime} / \phi_{2}
\end{aligned}
$$

(According to Equation $3.15, \phi_{2} \approx I^{\prime} /(1-a)$. ) given by:

The normalized coefficients of Equation $3.2, a_{g}$ and $1-a_{g}$, are

$$
\begin{aligned}
& a_{g}=\phi_{2} /\left(\phi_{2}-\phi_{1}\right) \\
& 1-a_{g}=-\phi_{1} /\left(\phi_{2}-\phi_{1}\right)
\end{aligned}
$$

If $\phi_{2} \gg \phi_{1}$, then:

$$
\begin{aligned}
& a_{g} \approx 1 \\
& 1-a_{g} \approx 0
\end{aligned}
$$


Assume that there are no pressed-wood products present, but that formaldehyde is injected at constant rate $e$ into a measuring chamber of volume $V$ containing gypsum wallboard with exposed area $A$.

4.1. Derivation of $\mathrm{k}_{\mathrm{d}}$

Setting $t=\infty$ in Equation 2.1:

$$
\left(I+k_{1} \cdot L\right) \cdot C_{\infty}=k_{2} \cdot L \cdot G_{\infty}+I^{\prime} \cdot C_{\infty}
$$

It follows that:

$$
I^{\prime}-I=k_{1} \cdot L-k_{2} \cdot L \cdot G_{\infty} / C_{\infty}
$$

Using Equation 2.3:

$$
\begin{aligned}
& I^{\prime}-I=k_{1} \cdot L-k_{2} \cdot L \cdot k_{1} /\left(k_{2}+k_{d}\right) \\
& I^{\prime}-I=k_{1} \cdot L \cdot k_{d} /\left(k_{2}+k_{d}\right)=K \cdot L \cdot k_{d} \\
& k_{d}=\left(I^{\prime}-I\right) /(K \cdot L)
\end{aligned}
$$

In order to determine $k_{d}$, $K$ must be calculated from experimentally derived quantities. The next section shows how to calculate $G_{\infty}$ and $K$.

\subsection{Determination of $G_{\infty}$ and $K$}

Assume that formaldehyde absorbed by wallboard is at equilibrium with airborne formaldehyde, and that the sources of formaldehyde are removed; $G=G_{\infty}$ and $C=C_{\infty}$. Then the absorbed formaldehyde will be removed either by exiting the room or by irreversible reaction in the wallboard.

$$
A \cdot G_{\infty}=\int_{0}^{\infty} I \cdot V \cdot C(t) d t+\int_{0}^{\infty} k_{d} \cdot A \cdot G(t) d t
$$

Ignoring the fast decay term, $C$ and $G$ are given by Equations 3.3 and 3.4, respectively. For desorption, the initial conditions $C_{0}$ and $G_{0}$ are replaced by $C_{\infty}$ and $G_{\infty}$, and the final conditions $C_{\infty}$ and $G_{\infty}$ are both replaced by 0. Substituting Equations 3.3-3.4 into Equation 4.6:

$$
\begin{aligned}
& A \cdot G_{\infty}=\left(I \cdot V \cdot C_{\infty} \cdot a+k_{d} \cdot A \cdot G_{\infty}\right) \cdot \int_{0}^{\infty} \exp \left(-\phi_{1} \cdot t\right) d t \\
& A \cdot G_{\infty}=\left(I \cdot V \cdot C_{\infty} \cdot a+k_{d} \cdot A \cdot G_{\infty}\right) / \phi_{1}
\end{aligned}
$$

Substituting Equation 4.5 for $k_{d}$ into Equation 4.8 and dividing by $V \cdot C_{\infty}$ :

$$
\mathrm{K} \cdot \mathrm{L}=\left(\mathrm{I} \cdot \mathbf{a}+\left(\mathrm{I}^{\prime}-\mathrm{I}\right)\right) / \phi_{1}
$$




$$
\begin{aligned}
& \mathrm{K}=\left(\mathrm{I} \cdot \mathrm{a}+\left(\mathrm{I}^{\prime}-\mathrm{I}\right)\right) /\left(\mathrm{L} \cdot \phi_{1}\right) \\
& \mathrm{G}_{\infty}=\mathrm{C}_{\infty} \cdot\left(\mathrm{I} \cdot \mathrm{a}+\left(\mathrm{I}^{\prime}-\mathrm{I}\right)\right) /\left(\mathrm{L} \cdot \phi_{1}\right)
\end{aligned}
$$

If $\mathrm{k}_{\mathrm{d}}=0 \mathrm{~h}^{-1}$ or equivalently, $\mathrm{I}^{\prime}=\mathrm{I}$, then $\mathrm{K}=\mathrm{I} \cdot \mathrm{a} /\left(\mathrm{L} \cdot \phi_{1}\right)$ and $\mathrm{G}_{\infty}=$ $\mathrm{I} \cdot \mathrm{a} \cdot \mathrm{C}_{\infty} /\left(\mathrm{L} \cdot \phi_{1}\right)$.

\subsection{Derivation of $\mathrm{k}_{1}$ and $\mathrm{k}_{2}$}

Substituting $\mathrm{k}_{1}{ }^{\prime}=\left(\mathrm{k}_{2}+\mathrm{k}_{\mathrm{d}}\right) \cdot \mathrm{K} \cdot \mathrm{L}$ (from Equation 2.3), $\delta=\phi_{2}-\phi_{1}$ and $\phi_{1}+\phi_{2}=\mathrm{I}+\mathrm{k}_{1}{ }^{\prime}+\mathrm{k}_{2}+\mathrm{k}_{\mathrm{d}}$ (from Equations 3.6 and 3.7 ) into equation 3.5 , and rearranging yields:

$$
\begin{aligned}
\left(\phi_{1}+\phi_{2}\right)^{2}-\left(\phi_{2}-\phi_{1}\right)^{2}=4 \cdot I \cdot k_{2}+4 \cdot k_{d} \cdot I+4 \cdot k_{d} \cdot k_{2} \cdot K \cdot L+4 \cdot k_{d} 2 \cdot K \cdot L(4.12) \\
4 \cdot \phi_{1} \cdot \phi_{2}=4 \cdot\left(k_{2}+k_{d}\right) \cdot\left(I+k_{d} \cdot K \cdot L\right)
\end{aligned}
$$

Substituting $I^{\prime}=I+k_{d} \cdot K \cdot L$ (Equation 4.4) into Equation 4.13:

$$
\phi_{1} \cdot \phi_{2}=\left(k_{2}+k_{d}\right) \cdot I^{\prime}
$$

Therefore:

$$
\mathrm{k}_{2}=\phi_{1} \cdot \phi_{2} / \mathrm{I}^{\prime}-\mathrm{k}_{\mathrm{d}}
$$

Substituting $\mathrm{k}_{2}+\mathrm{k}_{\mathrm{d}}=\phi_{1} \cdot \phi_{2} / \mathrm{I}^{\prime}$ (Equation 4.15) into equation 2.3:

$$
\mathrm{k}_{1}=\phi_{1} \cdot \phi_{2} \cdot \mathrm{K} / \mathrm{I}^{\prime}
$$

\section{EXPERIMENTAL PLAN FOR VERIFYING THE SORPTION MODEL}

\subsection{Derivation of the Model Constants by Chamber Tests}

The sorption model may be tested using a measuring chamber under controlled temperature and $\mathrm{RH}$, as illustrated by the preliminary experiment described in Figures 2-4 and in Appendix A. First, the gypsum wallboard should be conditioned at the test conditions of temperature and $\mathrm{RH}$ in a formaldehyde-free atmosphere. It should be verified that the chamber walls and air do not react with formaldehyde and that the wallboard does not emit any formaldehyde. Then, each specimen of wallboard should be tested over a range of values of $I$ commonly found in the field, from about $0.1 \mathrm{~h}^{-1}$ to over $1 \mathrm{~h}^{-1}$, to ensure that the model constants do not vary with $I$. Wallboard from several different manufacturers, and from different and the same lot from the same manufacturer should be tested at the same temperature and RH to study the variation of model constants over available products. 


\subsection{Test of Assumption of First-Order Sorption Kinetics}

The range of values of $\mathrm{C}_{\infty}$ over which wallboard absorption is first order, that is $G_{\infty}$ is proportional to $C_{\infty}$, may be obtained by using a range of values of e. Injecting formaldehyde at a low rate e into a measurement chamber containing a specimen of wallboard, permit $C$ to stabilize to $C_{\infty}$ and determine $G_{\infty}$. Keeping $I$ fixed, raise e and repeat the experiment. The first order assumption starts breaking down when an increase of e results in a smaller increase in $G_{\infty}$. Absorption becomes zero order when increasing $e$ has no further effect on $G_{\infty}$.

\subsection{Painted and Coated Gypsum Wallboard}

The sorption model should be tested for coated wallboard since wallboard is usually painted or covered by wallpaper. Latex paint, which is almost invariably used for painting wallboard, has been found to be ineffective as a formaldehyde emission barrier [19-20]. Therefore, one would expect that latex paint would not affect sorption kinetics. Formaldehyde sorption through more impermeable wallpapers and glues might be zero order.

\subsection{Temperature- and RH-dependence of Sorption}

If sorption is fairly uniform over all products, and occurs as predicted, the next step is to study the temperature- and $\mathrm{RH}$-dependence of sorption in order to make the model usable under field conditions. In addition, the variation of model constants with temperature and $\mathrm{RH}$ may give information about the nature of the binding sites and the binding reaction.

\subsection{Diffusion within Wallboard}

Repeating the experiment with two boards from the same lot stacked together can yield useful information on the diffusion of formaldehyde through wallboard. If a stack of boards absorbs more formaldehyde than a single board, then it can be concluded that formaldehyde can diffuse completely through a board. The time for diffusion through a board can be estimated. Another way of obtaining this kind of information is to use wallboard to separate the measuring chamber into two compartments whose only communication route is through the wallboard. Inject formaldehyde into one compartment while monitoring the concentration on both sides of the board, as was done by Kazakevics in characterizing the permeability of polymers to formaldehyde [21].

\subsection{Diffusion within Air}

The role of formaldehyde diffusion in air can be studied by varying the speed of a mixing fan in the measuring chamber. If absorption is 
limited by the availability of formaldehyde in the air next to the wallboard, then increasing the fan speed should increase the absorption rate by making more formaldehyde available. Similarly, if desorption is limited by excess formaldehyde in the air next to the wallboard, then increasing the fan speed should increase the desorption rate by dispersing the formaldehyde away from the board.

\subsection{Validation of Sorption Model in a House}

The sorption model may be validated in a house containing wallboard and pressed-wood products maintained at fixed temperature and $\mathrm{RH}$, as illustrated in Figure 1 and described in the experimental methods section below. The model should be validated for several different ratios of I to L, using several different "typical" loadings of pressed wood products, and at reasonable combinations of temperature and RH after the model is expanded to account for these.

\section{SUMMARY}

Gypsum wallboard formaldehyde sorption was studied in experiments performed in a prototype house and in an environmentally controlled measuring chamber. Sorption was shown to be biphasic in response to a change in the air exchange rate or to the removal of the formaldehyde source. Consistent with observations by other researchers, a rapid initial phase was followed by a slow phase lasting several days. A formaldehyde sorption model accounting for the observed biphasic concentration pattern was derived on the basis of mass balance. Experiments for testing the predictability of the model were described.

\section{ACKNOWLEDGMENT}

This project was supported by the U. S. Consumer Product Safety Commission.

\section{REFERENCES}

[1] National Research Council, "Formaldehyde and other aldehydes," National Academy Press, 1981, Washington.

[2] Versar, Inc., "Formaldehyde exposure in residential settings: sources, levels, and effectiveness of control options," prepared for U.S. Environmental Protection Agency, Springfield, VA, 1986.

[3] G. E. Myers, "Effect of ventilation rate and board loading on formaldehyde concentration: a critical review of the literature," Forest Prod. J. 34, 59-68, 1984.

[4] S. Fujii, T. Suzecki, and S. Koyagaehiro, "Study on liberated formaldehyde as renewal for JIS particleboard," Kenjai Shiken Joho $9,10-14,1973$. 
[5] J. J. Hoetjer, "Introduction to a theoretical model for the splitting of formaldehyde from composition board," Methanol Chemie Nederland, June 8, 1978.

[6] A. Berge, B. Milligaard, P. Haneto, and E. B.Ormstad, Holz als Rohund Werkstaff 1980, 38, 251-255.

[7] G. E. Myers and M. Nagaoka, "Formaldehyde emission: methods of measurement and effects of several particleboard variables," Wood Sci. 13, 140-150, 1981 .

[8] T. G. Matthews, T. J. Reed, B. J. Tromberg, K. W. Fung, C. V. Thompson, J. O. Simpson, and A. R. Hawthorne, "Modeling and testing of formaldehyde emission characteristics of pressed-wood products: report XVIII to the U. S. Consumer Product Safety Commission 1985," Oak Ridge National Laboratory report no. TM-9867, Oak Ridge, TN.

[9] B. Meyer and K. Hermanns, "Formaldehyde release from pressed wood products," pp. 101-116 in V. Turoski, Ed., "Formaldehyde analytical chemistry and toxicology," American Chemical Society, Washington, 1985.

[10] T. G. Matthews, K. W. Fung, B. J. Tromberg, and A. R. Hawthorne, "Impact of indoor environmental parameters on formaldehyde concentrations in unoccupied research houses," JAPCA 36, 1244-1249, 1986.

[11] A. R. Hawthorne and T. G. Matthews, "Models for estimating organic emissions from building materials: formaldehyde example," Atm. Environ. 21, 419-424, 1987.

[12] T. G. Matthews, A. R. Hawthorne, and C. V. Thompson, "Formaldehyde sorption and desorption characteristics of gypsum wallboard," Environ. Sci. and Technol. 21, 629-634, 1987.

[13] S. Silberstein, R. A. Grot, K. Ishiguro, and J. L. Mulligan, "Validation of models for predicting formaldehyde concentrations in residences due to pressed-wood products," JAPCA 38, 1403-1411, 1988 .

[14] T. Miyazaki, "Adsorption characteristics of $\mathrm{NO}_{2}$ by several kinds of interior materials," pp. 103-110 in "Proceedings of the third air quality international conference on indoor air quality and climate," vol. 4, Swedish Council for Building Research, Stockholm, 1984.

[15] S. Yamanaka, "Decay rates of nitrogen oxides in a typical Japanese living room," Environ. Sci. and Technol. 18, 566-570, 1984.

[16] B. P. Leaderer, S. Renes, P. Bluyssen, H. van de Loo, "Chamber studies of $\mathrm{NO}_{2}, \mathrm{SO}_{2}$, and RSP deposition rates indoors, "Proc. APCA Annual Meeting, 79th (Vol. 2), 86-38.3, 1986.

[17] T. Godish and B. Kanyer, "Formaldehyde source interaction studies," For. Prod. J. 35, 13-17, 1985.

[18] B. Meyer and K. Hermanns, "Formaldehyde indoor air problems," Proc. APCA Annual Meeting, 77th (Vol. 2), 84-35.2, 1984.

[19] A. R. Hawthorne, R. B. Gammage, T. G. Matthews, G. S. Blackman, T. C. Howell, and R. J. Allen, Oak Ridge National Laboratory report no. TM 7959, 1981, Oak Ridge, TN.

[20] R. A. Grot, S. Nabinger, and S. Silberstein, "Formaldehyde emissions from low-emitting pressed-wood products and the effectiveness of various remedial measures for reducing formaldehyde emissions," in preparation. 
[21] A. A. R. Kazakevics, "Studies on the reduction of formaldehyde emission from particleboard by polymers," Ph. D. thesis, University of Auckland, New Zealand, 1984.

[22] R. A. Grot, S. Silberstein, and Ishiguro, "Validation of models for predicting formaldehyde concentrations in residences due to pressed-wood products, phase I," National Bureau of Standards (now National Institute of Standards and Technology) interagency report 85-3255, Gaithersburg, MD, 1985.

[23] S. Silberstein, "Comparison of the chromotropic acid and pararosaniline methods for measuring formaldehyde concentrations of pressed-wood product emissions," Am. Ind. Hyg. Assoc. J., in press. 


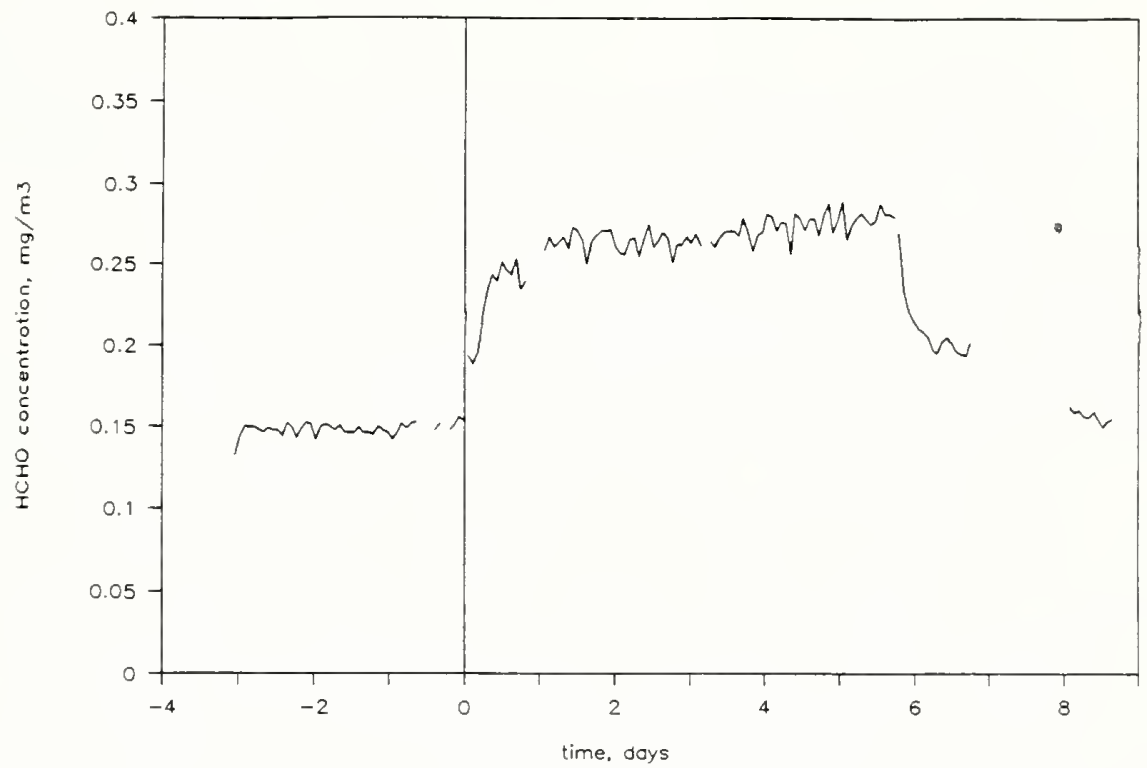

Figure 1. Measured formaldehyde concentration in a prototype house containing gypsum wallboard, particleboard flooring, medium-density fiberboard, and hardwood-plywood paneling. Before 0 days, the air exchange rate was $0.58 \mathrm{~h}^{-1}$. Between 0 and 5.75 days, the air exchange rate was $0.27 \mathrm{~h}^{-1}$. After 5.75 days, the air exchange rate was $0.75 \mathrm{~h}^{-1}$. The temperature was $23^{\circ} \mathrm{C}$ and the $\mathrm{RH}$ was 508 .

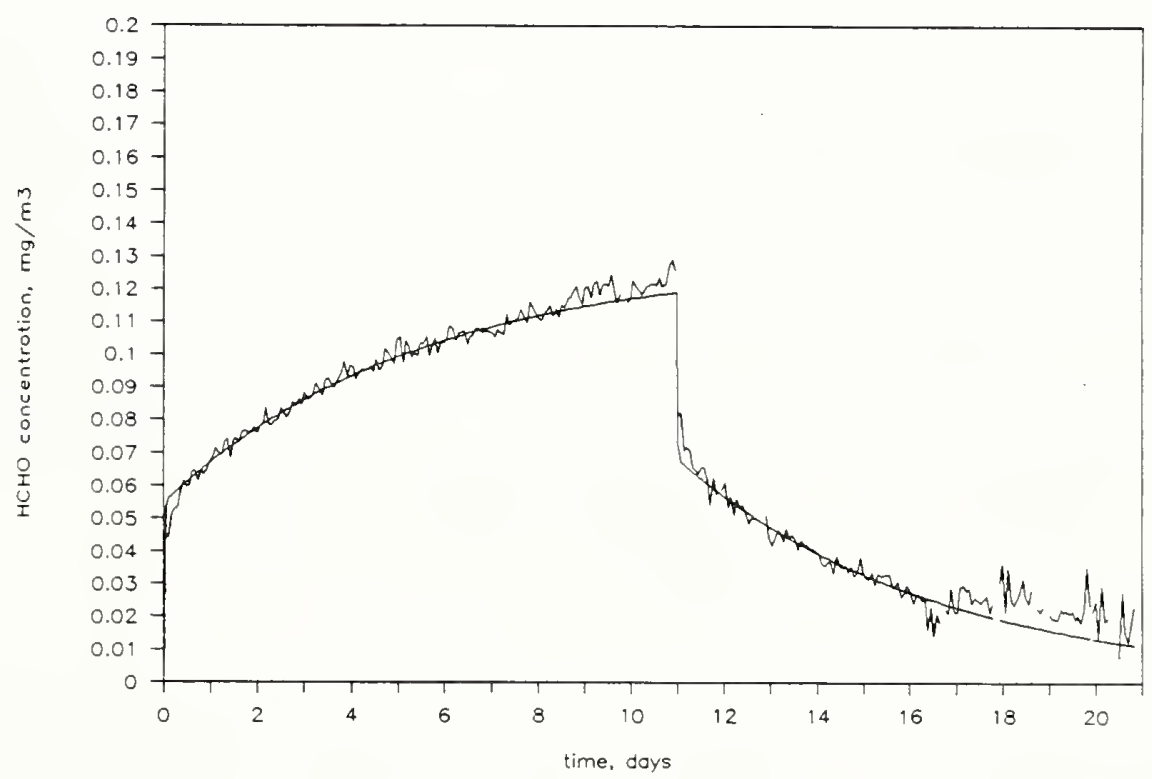

Figure 2. Measured and predicted formaldehyde concentration (jagged and smooth curves, respectively) in a medium-sized measuring chamber containing gypsum wallboard. Formaldehyde injection (absorption) began at 0 days. Injection ended and desorption began at 11.0 days. The temperature was $23^{\circ} \mathrm{C}$ and the $\mathrm{RH}$ was 508 . 


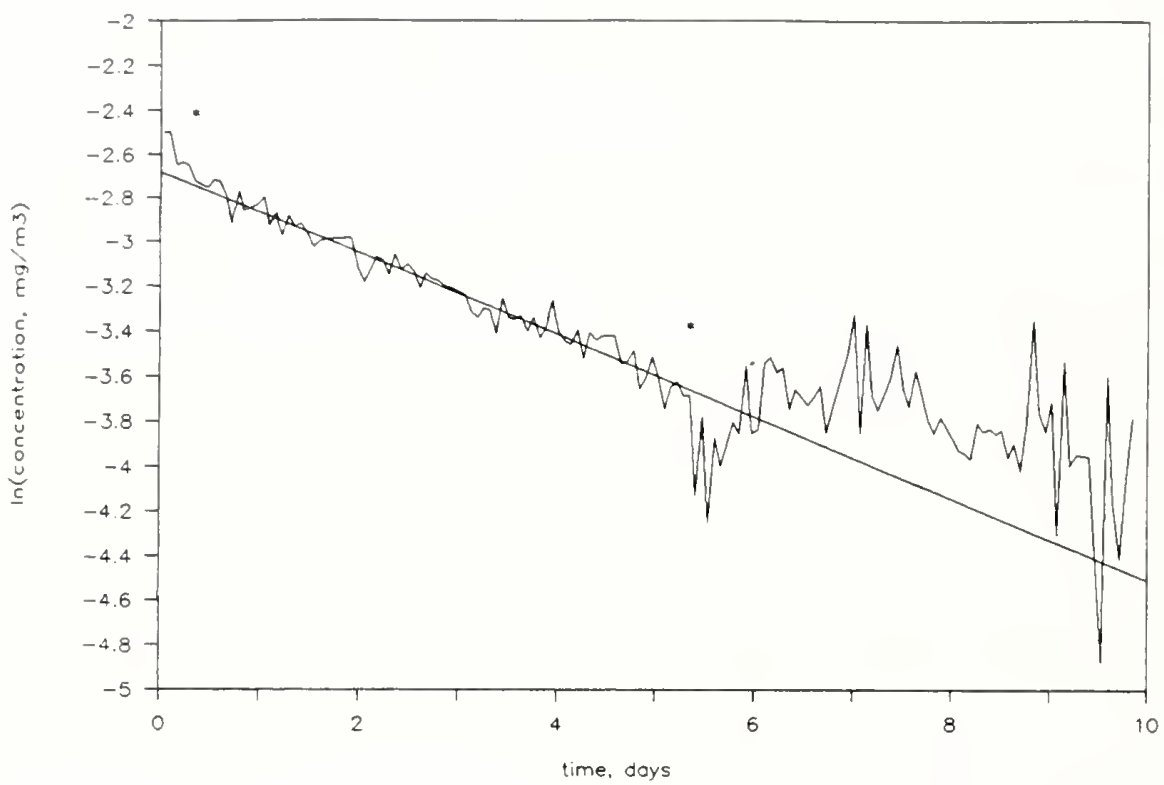

Figure 3. Logarithm of formaldehyde concentration as a function of time during formaldehyde desorption by gypsum wallboard (Figure 2). The straight line is the best-fit regression line for the data between the asterisks.

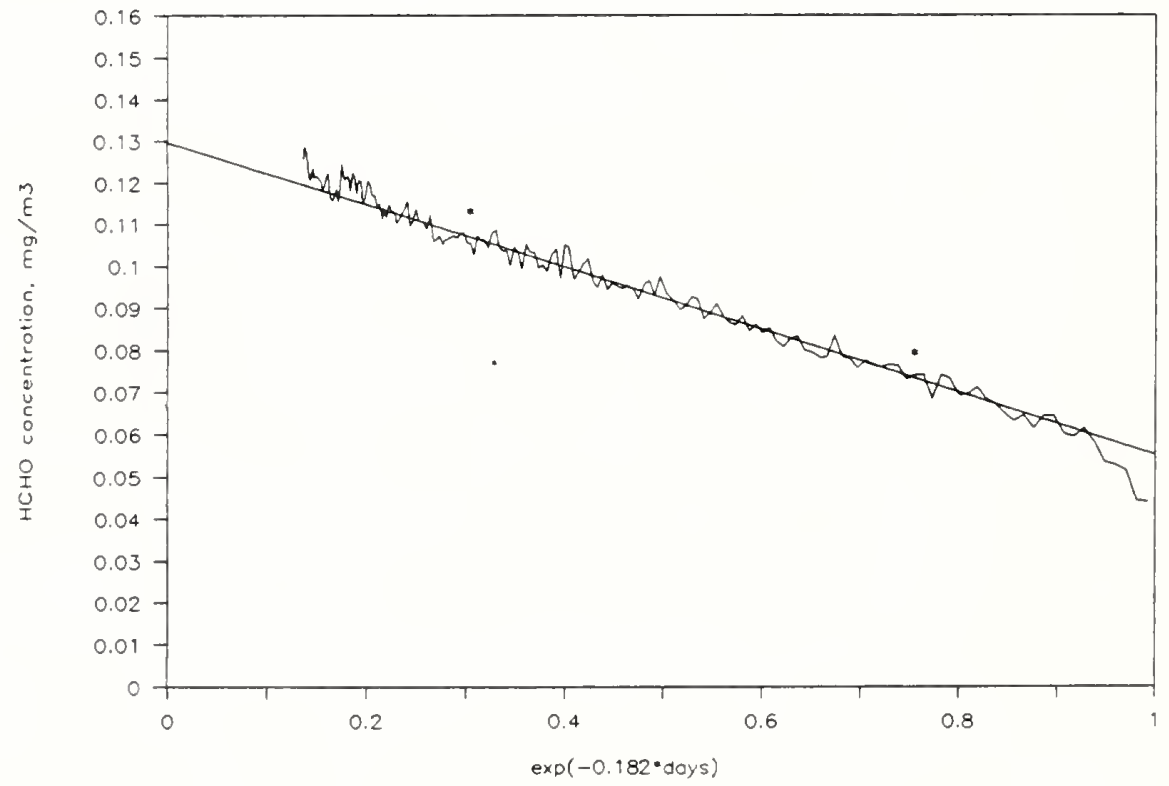

Figure 4. Formaldehyde concentration as a function of $\exp (-0.182 \cdot$ days $)$ during formaldehyde absorption by gypsum wallboard (Figure 2). The straight line is the best-fit regression line for the data between the asterisks. 


\section{APPENDIX A. METHODS USED IN THE PRELIMINARY EXPERIMENTS*}

This appendix describes the methods used in the preliminary experiments shown in Figures 1-2. Figure 1 shows qualitatively that the formaldehyde concentration in a prototype house containing gypsum wallboard and pressed-wood products responds in a biphasic manner to changes in the air exchange rate. Figure 2 compares measured and predicted formaldehyde concentrations in a medium-sized measuring chamber containing gypsum wallboard during formaldehyde injection and after injection ceased. The experiment confirms the biphasic concentration profile predicted by the sorption model.

\section{A.1. Prototype-House Studies}

The prototype house, designed to study combinations of pressed-wood products in a building resembling typical houses; the automated pararosaniline method for measuring formaldehyde concentrations; and the automated sulfur hexafluoride tracer decay method for measuring air exchange rates were described elsewhere $[13,22]$. The house was located in an environmental chamber maintained at $23^{\circ} \mathrm{C}$ and $50 \% \mathrm{RH}$ throughout the experiments. The interior dimensions of the prototype house were $3 \mathrm{~m} \times 6$ $\mathrm{m} \times 2.4 \mathrm{~m}$, for a volume of $45 \mathrm{~m}^{3}$. The two equal-sized rooms were connected by a doorway which was left open during testing. The floor, ceiling, and sidewalls were lined with a polyethylene vapor barrier.

The prototype house contained 6 specimens of particleboard flooring, 3 specimens of hardwood-plywood paneling from 2 different manufacturers on the walls, and 2 tables, each having 1 exposed surface of medium-density fiberboard (mdf). The walls were covered by gypsum wallboard; for exposed wallboard, $L=1.44 \mathrm{~m}^{-1}$.

Formaldehyde was sampled at the inlet, outlet, and the interior of each room. Figure 1 shows the formaldehyde concentration measured at the outlet. (The outlet concentration was approximately $0.01 \mathrm{mg} / \mathrm{m}^{3}$ greater than the concentration in one room and $0.01 \mathrm{mg} / \mathrm{m}^{3}$ lower than the concentration in the other room.)

The air exchange rate was determined using the tracer decay method. Sulfur hexafluoride was injected into the inlet air and sampled at the outlet.

\section{A.2. Measuring-Chamber Studies}

Gypsum wallboard sorption was studied in a "medium-sized" measuring chamber. The chamber, designed to measure individual pressed-wood products was described elsewhere $[13,22]$. The chamber was located inside an environmental chamber maintained at $23^{\circ} \mathrm{C}$ and $50 \% \mathrm{RH}$ throughout the experiments.

\footnotetext{
${ }^{*}$ Literature references are cited in the References section above.
} 


\section{A.2.1. Formaldehyde Generation}

Gas standards generators were used for calibrating the formaldehyde concentration analysis, and for injecting formaldehyde into the measuring chambers at known rates, also as described elsewhere [22-23]. For calibration, "span gas" containing about $0.1 \mathrm{mg} / \mathrm{m}^{3}$ formaldehyde was produced by heating polyoxymethylene permeation tubes at an oven temperature of $80^{\circ} \mathrm{C}$. The emission rate of the permeation tubes was determined by approximately monthly weighing at room temperature after removal from the oven. The mass loss rate was determined by linear regression analysis of time vs. mass. The relative error of the emission rate was estimated to be about 28 .

An oven temperature of $100^{\circ} \mathrm{C}$ was used for heating permeation tubes in order to inject chambers with formaldehyde. The tubes were weighed approximately daily at room temperature after removal from the oven. An emission rate of $0.313 \mathrm{mg} / \mathrm{h}$ was determined.

\section{A.2.2. Gypsum Wallboard Emission and Sorption Measurements}

A sample of new gypsum wallboard $(1.2 \mathrm{~m} \times 2.4 \mathrm{~m})$ was installed into the medium-sized chamber $(1.2 \mathrm{~m} \times 2.4 \mathrm{~m} \times 0.6 \mathrm{~m})$. The loading of wallboard was $1.64 \mathrm{~m}^{-1}$.

Formaldehyde absorption behavior of the wallboard was studied by monitoring formaldehyde concentration for 11 days while a gas standards generator injected formaldehyde into the measuring chamber. Desorption was studied by monitoring concentration for 10 days after injection ceased, without adjusting any of the air flows into, out of, or within the chamber.

\section{A.2.3. Predicting Formaldehyde Concentration from Sorption Measurements}

The desorption and absorption data of Figure 2 are presented in Figures 3 and 4 , respectively, in ways more convenient for parameter calculation. At the start of desorption ( $t=11.0$ days), $C_{0}$ was measured to be $0.12 \mathrm{mg} / \mathrm{m}^{3}$. Using the desorption data between the asterisks in Figure $3, \phi_{1}=0.182$ day $^{-1}=0.0076 \mathrm{~h}^{-1}$ and $\mathrm{a}=0.570$ were calculated by linear regression analysis of time after 11.0 days vs. $\ln (C(t))$, assuming and $C_{\infty}=0 \mathrm{mg} / \mathrm{m}^{3}$ (see Equation 3.3; the slope is $-\phi_{1}$ and the intercept is $\left.\ln \left(C_{0} \cdot a\right)\right)$. Early concentrations (to the left of the asterisks) were still influenced by $\phi_{2}$, and later concentrations (to the right of the asterisks) were too scattered for undetermined reasons. At the start of absorption, $C_{0}$ was measured to be $0 \mathrm{mg} / \mathrm{m}^{3}$. Assuming that $\phi_{1}=0.182$ day $^{-1}$, the absorption data between the asterisks in Figure 3 were used to calculate $C_{\infty}=0.13 \mathrm{mg} / \mathrm{m}^{3}$ and $\mathrm{a}=0.575$ by linear regression analysis of $\exp \left(-\phi_{1} \cdot t\right)$ vs. $C(t)$ (see Equation 3.3 ; the slope is $-a \cdot C_{\infty}$ and the intercept is $C_{\infty}$ ).

The determinations of "a" from the results of the desorption and absorption experiments are nearly identical. (However, calculating the value of "a" from absorption data does not require estimating $\mathrm{C}_{0}$, and is therefore probably more reliable, in general, than using desorption 
data.) The theoretical curves for $C$ given by Equation 3.1, calculated using $a=0.575, \phi_{1}=0.182 \mathrm{day}^{-1}, \mathrm{I}^{\prime}=1.3 \mathrm{~h}^{-1}$ (from Equation 3.9), and $\phi_{2}=3.1 \mathrm{~h}^{-1}$ (from Equation 3.13), are shown in Figure 2. The curves fit both absorption and desorption data very well. 


\begin{tabular}{|c|c|c|}
\hline \multirow{4}{*}{$\begin{array}{l}\text { NIST-114A } \\
\text { (REV. 3-89) }\end{array}$} & \multirow{2}{*}{$\begin{array}{l}\text { U.S. DEPARTMENT OF COMMERCE } \\
\text { NATIONAL INSTITUTE OF STANDARDS AND TECHNOLOGY }\end{array}$} & $\begin{array}{l}\text { 1. PUBUCATION OR REPORT NUMBER } \\
\text { NISTIR } 89-4028\end{array}$ \\
\hline & & $\begin{array}{l}\text { NISTIR 89-4028 } \\
\text { 2. PERFORMIAO ORQAMIZATION REPORT HUMBEA }\end{array}$ \\
\hline & \multirow[t]{2}{*}{ BIBLIOGRAPHIC DATA SHEET } & \\
\hline & & NOVEMBER 1989 \\
\hline
\end{tabular}

4. TITLE AND SUBTITLE

A Gypsum Wallboard Formaldehyde Sorption Model

5. AUTHOR(S)

Samuel Silberstein

6. PERFORMINO ORGANIZATION (IF JOINT OA OTHER THAN NIST, SEE INSTAUCTIONS)

U.S. DEPARTMENT OF COMMERCE

NATIONAL INSTITUTE OF STANDARDS AND TECHNOLOQY

GAITHERSBURG, MD 20899

7. CONTRACT/ORANT MUMBER

8. TYPE OF REPOAT AND PERIOD COVERED

9. SPONSORING ORGAMIZATION NAME AND COMPLETE ADDRESS (STREET, CITY, STATE, ZIP)

NIST

U. S. Department of Commerce

10. SUPPLEMEMTARY NOTES

DOCUMENT DESCRIBES A COMPUTER PAOGRAM; SF-185, FIPS SOFTWARE SUMMARY, IS ATTACHED.

11. ABSTRACT (A 200-WOAD OA LESS FACTUAL SUMMAAY OF MOST SIONIFICANT INFOAMATION. IF DOCUMENT INCLUDES A SIOMIFICANT BIBLIOQAAPHY OA LITERATUAE SURVEY, MENTION IT HERE.)

Gypsum wallboard was shown to absorb formaldehyde in a prototype house and in a measuring chamber, as reported previously by researchers at Oak Ridge National Laboratory (ORNL). Also as reported previously, formaldehyde concentrations attained equilibrium in two phases in response to a change in the air exchange rate or to the removal of the formaldehyde source. A rapid initial phase was followed by a slow phase lasting several days. A formaldehyde sorption model that accounts for the biphasic concentration pattern is presented here. Experiments for testing the predictability of the model are proposed.

12. KEY WORDS (6 TO 12 ENTRIES; ALPHABETICAL ORDER; CAPITALZE ONLY PROPER HAMES; AND SEPARATE KEY WORDS BY SEMICOLONS)

Formaldehyde; gypsum; indoor air quality; modeling; pollutant; sorption; source-sink; wallboard.

FOR OFFICIAL DISTRIBUTION. DO NOT RELEASE TO NATIONAL TECHNICAL INFORMATION SEAVICE (NTIS). ORDER FROM SUPERINTENDENT OF DOCUMENTS, U.S. GOVERMMENT PAINTINQ OFFICE, WASHINATOH, DC 20402. 


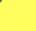


